\title{
Emergence of Clusters: Halos, Efimov States, and Experimental Signals
}

\author{
D. Hove,${ }^{1}$ E. Garrido, ${ }^{2}$ P. Sarriguren, ${ }^{2}$ D. V. Fedorov, ${ }^{1}$ H. O. U. Fynbo, ${ }^{1}$ A. S. Jensen, ${ }^{1}$ and N. T. Zinner ${ }^{1}$ \\ ${ }^{1}$ Department of Physics and Astronomy, Aarhus University, DK-8000 Aarhus C, Denmark \\ ${ }^{2}$ Instituto de Estructura de la Materia, IEM-CSIC, Serrano 123, E-28006 Madrid, Spain
}

\author{
(Received 21 April 2017; revised manuscript received 1 October 2017; published 31 January 2018)
}

\begin{abstract}
We investigate the emergence of halos and Efimov states in nuclei by use of a newly designed model that combines self-consistent mean-field and three-body descriptions. Recent interest in neutron heavy calcium isotopes makes ${ }^{72} \mathrm{Ca}\left({ }^{70} \mathrm{Ca}+n+n\right)$ an ideal realistic candidate on the neutron dripline, and we use it as a representative example that illustrates our broadly applicable conclusions. By smooth variation of the interactions we simulate the crossover from well-bound systems to structures beyond the threshold of binding, and find that halo configurations emerge from the mean-field structure for three-body binding energy less than $\sim 100 \mathrm{keV}$. Strong evidence is provided that Efimov states cannot exist in nuclei. The structure that bears the most resemblance to an Efimov state is a giant halo extending beyond the neutroncore scattering length. We show that the observable large-distance decay properties of the wave function can differ substantially from the bulk part at short distances, and that this evolution can be traced with our combination of few- and many-body formalisms. This connection is vital for interpretation of measurements such as those where an initial state is populated in a reaction or by a beta decay.
\end{abstract}

DOI: 10.1103/PhysRevLett.120.052502

Introduction.-Constituent particles of $N$-body nuclear structures are usually approximated as pointlike nucleons where the intrinsic degrees of freedom are frozen. The simplest structure beyond the deuteron is the uncorrelated average structure from the mean-field assumption of antisymmetrized product wave functions [1-5]. Often correlations are decisive and require special treatment [6-13].

Nuclear structures can vary from spherical mean-field properties, over collective deformations and a variety of other correlations, to bound nuclear clusters each in (almost) inert subsystems [7,14-16]. Very crudely, we can say that nuclei around beta stability are fairly well described by self-consistent mean-field calculations while the approach to the nucleon driplines produces two- and three-body halo structures $[17,18]$. For excited states at energies close to threshold for cluster separation, the corresponding clusterization is strongly favored [19].

If the clusters, in addition to weak binding, have a sufficiently large $s$-wave scattering length, the emergence of Efimov physics is theoretically possible in nuclear systems [20-26]. More recently, the neutron rich end of the calcium isotope chain was suggested as a likely candidate for Efimov physics [27]. However, interest in Efimov physics or Efimov states extends much beyond nuclear physics and covers diverse areas including cold atomic gases [28], clusters of helium [29], and lately also exotic photon systems [30].

For heavy systems traditional clusterized few-body techniques seem problematic, as they tend to neglect the internal structure of the clusters. In a newly designed model the core with many nucleons is treated in a mean-field approximation and the valence particles with few-body techniques [31]. This model allows us to investigate the emergence of halo and Efimov states close to the neutron dripline, using ${ }^{72} \mathrm{Ca}$ as the test case. This choice is very well suited for several reasons: mean-field computations find that ${ }^{70} \mathrm{Ca}$ is a spherical closed shell dripline nucleus, ${ }^{72} \mathrm{Ca}$ is mean-field unbound, but a stable Borromean nucleus in three-body calculations. The $s$ state near the Fermi level provides optimal conditions for pronounced halo structures and possibly Efimov states.

The purpose of the present Letter is threefold: (i) to discuss the emergence of halos, (ii) examine the possible existence of Efimov-states for realistic effective interactions, and (iii) make a connection between short- and large-distance quantum solutions.

Model ingredients.-Our starting point is an $A+2$ nucleon system viewed as a core with mass number $A$ and two valence nucleons. We assume two- and three-body interactions, $V_{i j}$ and $V_{i j k}$, acting between nucleons. Our general Hamiltonian is

$$
H=\sum_{i=1}^{A+2} T_{i}-T_{c m}+\sum_{i<j}^{A+2} V_{i j}+\sum_{i<j<k}^{A} V_{i j k}+V_{3 b},
$$

where $T_{i}$ and $T_{c m}$ are kinetic energy operators for the $i^{\prime}$ th nucleon and the total $A+2$ system, respectively, and $V_{3 b}$ is the three-body interaction between the valence neutrons and the core particles. Central to our method is the choice of Hilbert space and the corresponding interactions. We shall use Skyrme forces and wave functions of the form 


$$
\Psi=\mathcal{A}\left[\phi_{c}\left(\boldsymbol{r}_{1}, \ldots, \boldsymbol{r}_{A}\right) \phi_{3}\left(\boldsymbol{r}_{v_{1}}, \boldsymbol{r}_{v_{2}}\right)\right],
$$

where $\phi_{c}$ is the Slater determinant of core nucleons with spin and space coordinates, $\left(\boldsymbol{r}_{1}, \ldots, \boldsymbol{r}_{A}\right), \phi_{3}$ is the threebody wave function with nucleon-core relative coordinates, $\left(\boldsymbol{r}_{v_{1}}, \boldsymbol{r}_{v_{2}}\right)$, and $\mathcal{A}$ is the antisymmetrization operator.

Equation (2) exhibits how we combine mean-field treatment of the core nucleons with few-body treatment of the two relative degrees of freedom. We emphasize that the effective interactions determining $\phi_{c}$ depends on $\phi_{3}$ and vice versa. The detailed formulations are presented in the Supplemental Material [32].

The three-body equation is solved using the hyperspherical adiabatic expansion method [20]. We exclude the core-occupied Pauli forbidden states either by removal of the associated hyperspheric states or by using phase equivalent potentials [34]. The final step is solving a coupled set of hyperradial equations, where the simplest hyperradial equations for extreme halos can be written as

$$
\left(-\frac{\partial^{2}}{\partial \rho^{2}}-\frac{\xi^{2}(\rho)+1 / 4}{\rho^{2}}+v_{\text {short }}(\rho)-\tilde{\epsilon}\right) f(\rho)=0,
$$

where $\rho$ is the hyperradius [20], $f(\rho)$ is the dominating radial wave function, and $v_{\text {short }}(\rho)$ and $\tilde{\epsilon}$ are reduced shortrange interaction and three-body energy. The parameter $\xi(\rho)$ is obtained from solving the angular part of the threebody equations. For very large $s$-wave scattering lengths $\xi$ is constant over a substantial $\rho$ interval, where Efimov states might exist. Energies and mean square radii of neighboring solutions are related through the scaling, $s^{2}=\exp (2 \pi / \xi)$. The $\rho$ dependence of this parameter is then of crucial importance for the occurrence of halos and existence of Efimov states.

Why ${ }^{72} \mathrm{Ca}\left({ }^{70} \mathrm{Ca}+n+n\right)$. - The ideal system is a spherical, closed shell, dripline nucleus. Our mean-field calculations with the SLy4 parametrization [35] predict that ${ }^{70} \mathrm{Ca}$ is bound while ${ }^{72} \mathrm{Ca}$ is unbound. This is in general agreement with other mean-field calculations of various types $[19,36]$. However, ${ }^{72} \mathrm{Ca}\left({ }^{70} \mathrm{Ca}+n+n\right)$ is bound in a threebody calculation where the two-body subsystems are unbound. Thus, ${ }^{72} \mathrm{Ca}$ is a Borromean nucleus at the neutron dripline within our model and a perfect case for the method with a mean-field core calculation.

Seeing that Skyrme interaction parameters are mainly fitted to the properties of experimentally well-known nuclei, it is not surprising that the predictions of the various Skyrme parametrizations differ when dealing with exotic nuclei. This is particularly true for the prediction of the neutron-dripline isotopes that may change significantly depending on the interaction. For $\mathrm{Ca}$ isotopes one finds neutron driplines ranging from $A=68-76$ [19].

A coupled cluster model instead predicts the neutron dripline to be at ${ }^{60} \mathrm{Ca}$ [27]. The difference is apparently that the $g_{9 / 2}$ level is much higher in these calculations, and the $s_{1 / 2}$ level is the first unoccupied level in ${ }^{60} \mathrm{Ca}$.
The three-body part with our method would be similar for both ${ }^{62} \mathrm{Ca}$ and ${ }^{72} \mathrm{Ca}$, deviating only by the piece of the neutron-core interaction arising from the filled $g_{9 / 2}$ levels. The essential $s_{1 / 2}$ level is unoccupied in both models. Therefore, the discussion about appearance of halos and Efimov states would be the same. Likewise, a deformed core would not affect any conclusions drawn, as it firmly established that a deformed core surrounded by $s$-wave neutrons would produce halo structure provided the binding energy is sufficiently small [37-39]. The proximity of the dripline is the decisive property, since approximate neutron-core decoupling, along with weak binding and large spatial extension, is then most likely. We shall vary the interactions and investigate the emergence of halo structures and the possible existence of Efimov states. Specifically, the global Skyrme parameters, usually denoted $t_{\{0-3\}}$, are scaled, such that $t_{i} \rightarrow S t_{i}$.

Basic two-body properties.-We study how cluster structure emerges when neutrons are added and the dripline approached. We simulate the dripline approach by scaling of the globally determined Skyrme parameters for the same number of nucleons.

The crucial quantity for the appearance of halos and Efimov states is the $n$-core $s$-wave interaction which must have a bound, virtual, or resonance state close to zero binding. The present mean-field plus three-body method folds this two-body interaction with the calculated core structure. The resulting neutron-core scattering length $a$ is shown for ${ }^{70} \mathrm{Ca}+n$ in Fig. 1 as a function of the overall scaling of the Skyrme parameters for both the Sly4 and the $\mathrm{SkM}^{*}$ [40] parametrizations.

The thresholds for $s$-wave binding, $|a|=\infty$, are placed on top of each other for the two Skyrme interactions. The sign change of the scattering length is very fast for the $\mathrm{SkM}^{*}$ interaction where only few points are obtained.

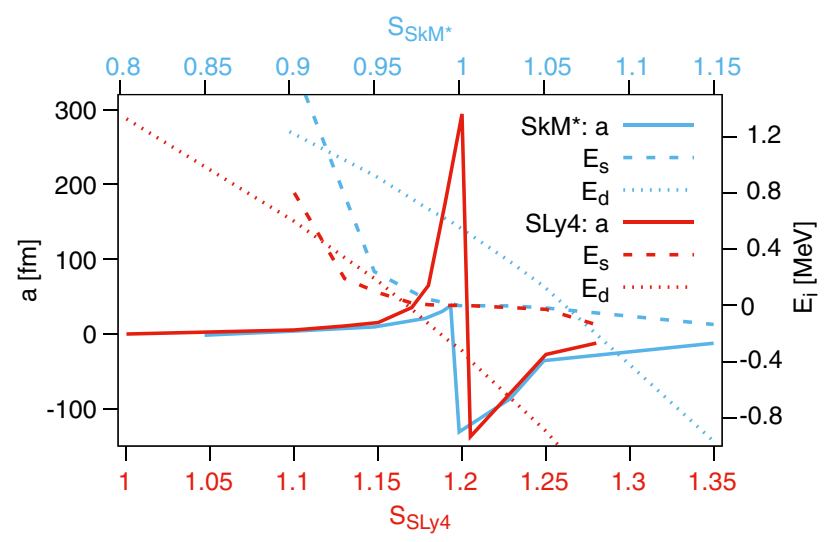

FIG. 1. The $s$-wave $n$-core scattering length $a$ (solid curves) as a function of the scaling of the Skyrme parameters $S$. The virtual energy of $s_{1 / 2}$ (dashed curves) is $\hbar^{2} /\left(2 \mu a^{2}\right)$, where $\mu$ is the reduced mass, and it is included along with the $d_{5 / 2}$ energy (dotted curves). Results are given for both the SLy4 (red curves) and $\mathrm{SkM}^{*}$ (blue curves) force. 
The estimated energy of the related virtual $s$ state is also shown as a measure of the distance from the threshold. The same pattern with $s$ - and $d$-wave crossings is found for both Skyrme interactions.

The properties of the $d$ level are the same, due to the centrifugal barrier, for energies slightly above and below the threshold of zero binding. If the $d$ level is much deeper the neutron dripline would be shifted from ${ }^{70} \mathrm{Ca}$ to ${ }^{76} \mathrm{Ca}$ corresponding to the 6 neutrons in the full $d_{5 / 2}$ shell. In this context, the results from these Skyrme interactions demonstrate the robustness of our conclusions.

Two-neutron halos.-Halo structures are characterized by very small binding energy and large radius which can be achieved most easily with high neutron occupancy of valence $s$ waves $[38,39]$. Scaling the Skyrme interaction or varying the three-body potential can both change the structure from confined to spatially extended two-neutron halo configuration. One complication in the present example is that the short-distance ground state configuration is dominated by $n$-core $d$ waves. Only the large-distance tail outside the $d$-wave centrifugal barrier is then expected to be $s$-wave dominated.

The simplest descriptive structure properties, related to spatial extension, are average distances. In Fig. 2 we exhibit rms distances as a function of the three-body energy below two-body threshold, obtained by adjusting the three-body potential, for three different values of $S$.

The average distance between the valence particles (solid curves) and between the valence particles and the core particles (dashed curves) is substantially larger than the corresponding average distances between the nucleons inside the core (dotted curves). This difference reflects that the two neutrons are among the highest-lying occupied levels [32]. The average separation is larger than the core radius, $\simeq 5 \mathrm{fm}$, revealing that they are spatially extended for these relatively small binding energies. Decreasing the binding energy towards zero leaves the nucleon-nucleon distances inside the core unchanged while the valence neutrons move away. Halo configurations emerge clearly for the two Borromean cases (red and blue curves) around a

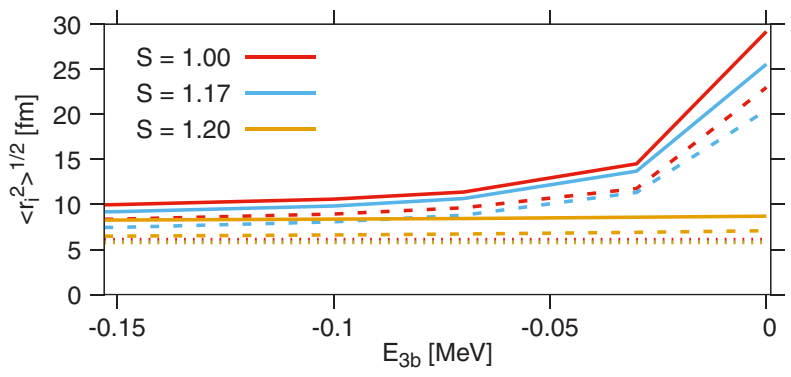

FIG. 2. rms distances as functions of the three-body energy, $E_{3 b}$. The two-body systems distances are valence $n-n\left\langle r_{n, n}^{2}\right\rangle^{1 / 2}$ (solid), valence $n$-core $\left\langle r_{c, n}^{2}\right\rangle^{1 / 2}$ (dashed), and core $n-n\left\langle r_{c, c}^{2}\right\rangle^{1 / 2}$ (dotted) radius for three different $S$ values. two-neutron binding of $100 \mathrm{keV}$ where the valence neutron distance increases rather abruptly.

The third case with $S=1.20$ in Fig. 2 would produce a bound two-body $d_{5 / 2}$ state with an energy approached by the adiabatic potential at large distance. Decreasing the binding energy towards zero with respect to this threshold populates $d$ waves and the rms distances remain finite as the $d$ waves prohibit spatially extended halo configurations. This fact shows that the appearance of the halo structure when approaching the dripline is not an inherent characteristic of the method, but a phenomenon that requires some particular conditions accounted for by the method itself.

The variation of $s$ - and $d$-wave contributions can be seen in Fig. 3 as functions of $\rho$, with $S=1.0$ (left) and $S=1.17$ (right). The three-body interaction is adjusted to give the quoted binding energies. The partial wave decomposition in Fig. 3 reveals that the $s$-wave tails (red curves) strongly increase with decreasing binding energy. The $s$-wave content is determined by the contributing lowest-lying adiabatic potentials, which in turn inherit the character of the lowestlying $n$-core single-particle structure. As a result the $s$-wave contribution is smaller for the scaled Skyrme interaction where the $d$-wave energy is closer to zero. Thus, the largedistance tails of the wave functions may change characteristics by fine-tuning the Skyrme interaction.

The traditional twofold criteria for the formation of halos is a very weakly bound two-body $s$ (or possibly $p$ ) state. From Fig. 2 we see how a weak binding, in the present nuclear case less than about $100 \mathrm{keV}$, leads to an extended state. However, the second part of the condition is seen to be more complicated from Fig. 3, where even a state with a dominating $d$ wave can lead to an extended halo structure provided a slight $s$-wave tail exists.

Halo formation is also possible in excited states which are less stable and often very fragile. The decisive properties are still small binding energy and dominating $s$-wave structure. States built on the second adiabatic potential are then tempting to study, since they are dominated by $s$ waves.

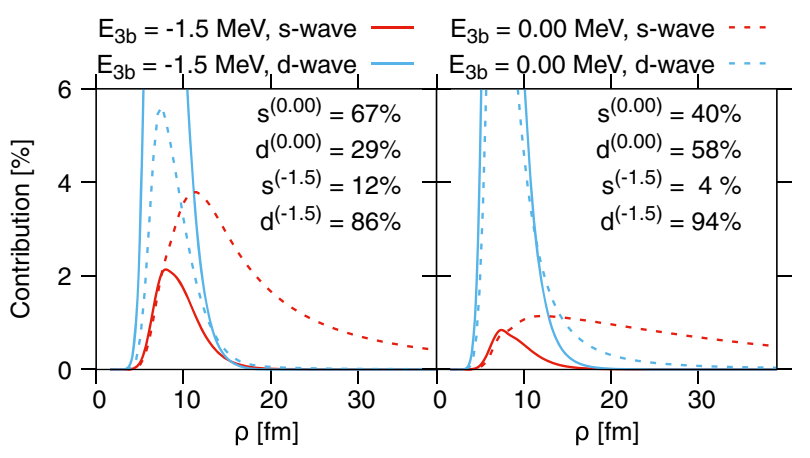

FIG. 3. The partial wave contributions from $s$ (red) and $d$ waves (blue) for $n$ core as functions of hyperradius for $S=1.0$ (left), and $S=1.17$ (right). Full and dashed lines indicate a three-body energy of -1.5 and $0.00 \mathrm{MeV}$, respectively. The total contribution after integration over $\rho$ is also given. 
Unfortunately, the $d_{5 / 2}$ state is then bound and excited three-body states built on the second adiabatic potential are discrete states on top of the two-body continuum from the first adiabatic potential. The states are therefore much more difficult to calculate, but still well defined and possible to obtain as demonstrated in Fig. 4. There is an overall similarity to the ground state results in Fig. 2, except that all distances are now larger. There is, however, a significant qualitative difference for $S=1.20$. Instead of remaining finite at threshold the valence related distances both increase enormously over the last $50 \mathrm{keV}$.

Efimov states.-This fast increase in size discussed in the previous section is the buildup of a halo, or theoretically the beginning of an Efimov state. In contrast to halos, for an Efimov state to appear it is not sufficient to have a small two-neutron separation energy. A very large $s$-wave scattering length $a$ is also required, which is mathematically known to produce an $1 / \rho^{2}$ potential of the form in Eq. (3) with $\xi^{2}>0$ and constant for distances much smaller than $a$. The convergence is extremely slow and only reached when $\rho$ is several orders of magnitude smaller than $a$, but still much larger than the light-light scattering length, which here is roughly $20 \mathrm{fm}$. This can only be achieved when $a$ is many orders of magnitude larger than 20 , which is very unrealistic for nuclei.

But let us assume the precursor of the Efimov series is obtained with a modest size of at least the range of the core potential, $r_{0}$. The Efimov scaling $s$ then predicts the radius of the next state to be $s$ times larger. We find $s \simeq 15$ $[18,22,41]$ from an assumption of three simultaneously large scattering lengths. This assumption is inherently true for zero-range models. However, this assumes that the neutron-neutron scattering length is much larger than both $r_{0}[18,41]$, and the size $s r_{0}$ of the first Efimov state. Since the neutron-neutron scattering length is around $20 \mathrm{fm}$, this state extends into the region of only two large scattering lengths. In this case, the scaling is determined by [22]

$$
\xi \cosh (\xi \pi / 2) \sin (2 \phi)=2 \sinh [\xi(\pi / 2-\phi)],
$$

with $\phi=\tan ^{-1}\left[\sqrt{\left(m_{c}\left(m_{c}+2 m_{n}\right)\right.} / m_{n}\right]$, where $m_{c / n}$ are the core and neutron masses. For ${ }^{72} \mathrm{Ca} \xi \simeq 0.01035$, which leads to an absurdly large scaling of $s \sim 10^{131}$.

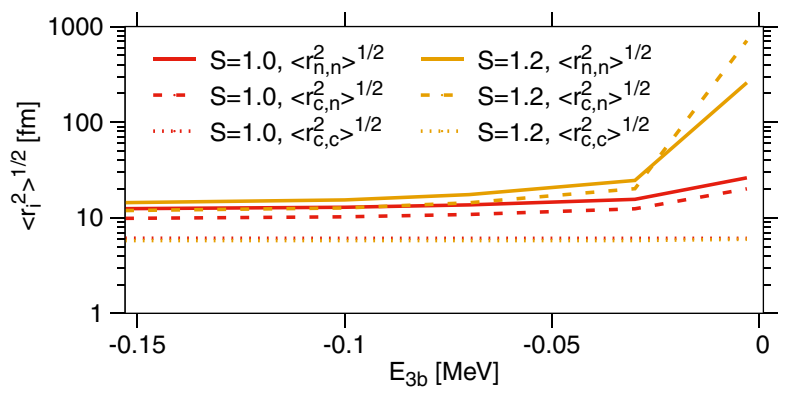

FIG. 4. The same as Fig. 2, on a logarithmic scale, for the first excited state with $S=1.0$ (red) or $S=1.20$ (orange).
The hypothetical first Efimov state must then be between $15 r_{0} \simeq 75-90 \mathrm{fm}$ (three large scattering lengths) and $10^{131} r_{0}$ (two large scattering lengths) where even the lower limit is much larger than $20 \mathrm{fm}$. This implies that this theoretical Efimov state must be located at distances several orders of magnitude larger than 75-90 fm, where it cannot exist due to outside influences. Thus there is no room for an Efimov state with these scaling properties. However, this does not mean that a pronounced halo state also is prohibited for a moderate scattering length of $300 \mathrm{fm}$. This discussion is very general and applies to all nuclei, including the sometimes proposed "nuclear Efimov candidates" such as ${ }^{62} \mathrm{Ca}$ [27] where $\xi \simeq 0.01205$ and $s \sim 10^{113}$, and also ${ }^{11} \mathrm{Li}$ [22] where $\xi \simeq 0.07382$ results in a scaling of $s \sim 10^{18}$, which is less extreme, but still enormous.

It is not surprising that Efimov states cannot appear, with a reasonable scaling, given that the system necessarily consists of one heavy and two light clusters with a relative small scattering length. A series expansion of Eq. (4) leads to

$$
|\xi| \approx \frac{4 m_{\text {light }}}{\sqrt{3} \pi m_{\text {heavy }}} .
$$

The exponential dependence on mass ratio effectively excludes the possibility of nuclear Efimov states.

Measurement interpretation.-The final state, singleparticle energy distribution is to a large extent dictated by the scattering lengths [23]. The single-particle energy distributions are also the simplest nontrivial observables to investigate, where a pertinent question is which information can be extracted from such a measurement. This can also be formulated by asking which path did the particles take before reaching the detectors, and perhaps even what was the decaying bulk structure located at small distances. This formulation is usually not meaningful in quantum mechanics where we have to be satisfied with a probability description for each path and initial state. However, the calculated wave function relates the observable large- and short-distance bulk properties.

In Fig. 5 the single-particle energy distributions $[42,43]$ are seen for a halo state with a total three-body energy at or

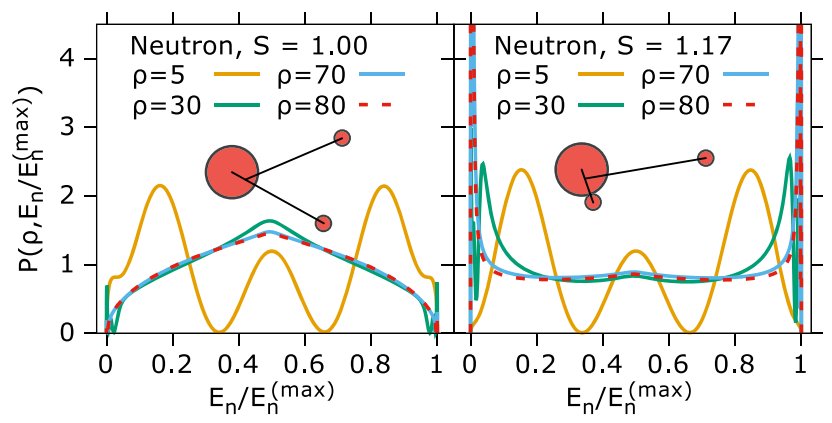

FIG. 5. The single-particle energy distributions for the neutron at $\rho=5,30,70$, and $80 \mathrm{fm}$, for $S=1.0$ (left) and $S=1.17$ (right). Schematic illustrations of the large distance configurations are also included. 
very slightly above zero. This is shown for $S=1.0$, corresponding to the last point on the red curve in Fig. 2, and for $S=1.17$, corresponding to the last point on the blue curve. In both cases the probability at around $\rho=5 \mathrm{fm}$ shows an oscillating distribution with three peaks. As $\rho$ increases these oscillations are smoothed out and the observable energy distributions appear at large distances. For $S=1.0$ the neutrons have an extended flat symmetric peak at half the maximum energy. This corresponds to a configuration with both the two neutrons moving away from the core. The pattern is typical for decay directly into the continuum without an intermediate stepping stone as in sequential decay.

For $S=1.17$ the initial configuration is very similar, but it evolves radically differently. From the three peak structure it transitions into a structure with one of the neutrons close to the core, while the other moves away, as encouraged by a $d_{5 / 2}$ level close to zero energy. This structure is then a signal of dominating two-body attractions between the unequal particles. Such distributions are typical for sequential decay via the resonance or bound state of the subsystem, here the $n$-core $d$ state.

As seen, the detectable large-distance distribution can be completely different from the short-distance properties. The $s$-wave dominance at large distance is necessary to produce a halo configuration, while other partial waves may be present in the short-distance bulk part. Thus, to learn about the short-distance structure we have to exploit other observables or be content with theoretically tracing the evolution of the wave function.

Conclusion.-A new method is applied to investigate how nuclear halos are formed by clusterization from a mean-field background of bound nucleons. The existence of a slight, long-distance, $s$-wave tail is found to be sufficient for the formation of halos even when dominated by other spatially confined states. Strong evidence that Efimov states cannot exist in nuclei for one heavy and two light particles is provided. Finally, we show how largedistance observables can differ qualitatively from bulk properties at small distances, and how the evolution of the wave function can be traced.

This work was funded by the Danish Council for Independent Research DFF Natural Science and the DFF Sapere Aude program. This work has been partially supported by the Spanish Ministerio de Economia y Competitividad under Project No. FIS2014-51971-P.

[1] D. Vautherin and D. M. Brink, Phys. Rev. C 5, 626 (1972).

[2] J. Dobaczewski, J. Phys. G 43, 04LT01 (2016).

[3] T. Niksić, D. Vretenar, and P. Ring, Prog. Part. Nucl. Phys. 66, 519 (2011).

[4] D. Vautherin, Phys. Rev. C 7, 296 (1973).

[5] M. Bender, P.-H. Heenen, and P.-G. Reinhard, Rev. Mod. Phys. 75, 121 (2003).
[6] B. R. Barrett, P. Navrátil, and J. P. Vary, Prog. Part. Nucl. Phys. 69, 131 (2013).

[7] E. Epelbaum, H.-W. Hammer, and U.-G. Meissner, Rev. Mod. Phys. 81, 1773 (2009).

[8] J. Carlson, S. Gandolfi, F. Pederiva, S. C. Pieper, R. Schiavilla, K. E. Schmidt, and R. B. Wiringa, Rev. Mod. Phys. 87, 1067 (2015).

[9] H. Hergert, S. K. Bogner, T. D. Morris, A. Schwenk, and K. Tsukiyama, Phys. Rep. 621, 165 (2016).

[10] A. Ono, H. Horiuchi, T. Maruyama, and A. Ohnishi, Prog. Theor. Phys. 87, 1185 (1992).

[11] H. Feldmeier and J. Schnack, Prog. Part. Nucl. Phys. 39, 393 (1997).

[12] G. Hagen, T. Papenbrock, D. J. Dean, and M. Hjorth-Jensen, Phys. Rev. C 82, 034330 (2010).

[13] Y. C. Tang and M. LeMere, Phys. Rep. 47, 167 (1978).

[14] W. Leidemann and G. Orlandini, Prog. Part. Nucl. Phys. 68, 158 (2013).

[15] G. Hagen, T. Papenbrock, M. Hjorth-Jensen, and D. J. Dean, Rep. Prog. Phys. 77, 096302 (2014).

[16] S. Liebig, U.-G. Meissner, and A. Nogga, Eur. Phys. J. A 52, 103 (2016).

[17] A. S. Jensen and K. Riisager, Phys. Lett. B 480, 39 (2000).

[18] T. Frederico, A. Delfino, Lauro Tomio, and M. T. Yamashita, Prog. Part. Nucl. Phys. 67, 939 (2012).

[19] J. Erler, N. Birge, M. Kortelainen, W. Nazarewicz, E. Olsen, A. M. Perhac, and M. Stoitsov, Nature (London) 486, 509 (2012).

[20] E. Nielsen, D. V. Fedorov, A. S. Jensen, and E. Garrido, Phys. Rep. 347, 373 (2001).

[21] D. V. Fedorov and A. S. Jensen, Phys. Rev. Lett. 71, 4103 (1993).

[22] A. S. Jensen and D. V. Fedorov, Europhys. Lett. 62, 336 (2003)

[23] E. Garrido, D. V. Fedorov, and A. S. Jensen, Phys. Rev. Lett. 96, 112501 (2006).

[24] V. Efimov, Phys. Lett. 33B, 563 (1970).

[25] I. Mazumdar and V. S. Bhasin, Phys. Rev. C 56, R5(R) (1997).

[26] I. Mazumdar, V. Arora, and V. S. Bhasin, Phys. Rev. C 61, 051303(R) (2000).

[27] G. Hagen, P. Hagen, H.-W. Hammer, and L. Platter, Phys. Rev. Lett. 111, 132501 (2013).

[28] J. P. D'Incao, arXiv:1705.10860.

[29] M. Kunitski, S. Zeller, J. Voigtsberger et al., Science 348, 551 (2015).

[30] M. J. Gullans, S. Diehl, S. T. Rittenhouse, B. P. Ruzic, J. P. D'Incao, P. Julienne, A. V. Gorshkov, and J. M. Taylor, Phys. Rev. Lett. 119, 233601 (2017).

[31] D. Hove, E. Garrido, P. Sarriguren, D. V. Fedorov, H. O. U. Fynbo, A. S. Jensen, and N. T. Zinner, Phys. Rev. C 95, 061301(R) (2017).

[32] See Supplemental Material at http://link.aps.org/ supplemental/10.1103/PhysRevLett.120.052502 for theoretical derivation of our method and specification of interactions, which includes Ref. [33].

[33] E. Garrido and E. Moya de Guerra, Nucl. Phys. A650, 387 (1999).

[34] E. Garrido, D. V. Fedorov, and A. S. Jensen, Nucl. Phys. A650, 247 (1999). 
[35] E. Chabanat, P. Bonche, P. Haensel, J. Meyer, and F. Schaeffer, Nucl. Phys. A635, 231 (1998).

[36] M. Bhattacharya and G. Gangopadhyay, Phys. Rev. C 72, 044318 (2005).

[37] T. Misu, W. Nazarewicz, and S. Berg, Nucl. Phys. A614, 44 (1997).

[38] A. S. Jensen, K. Riisager, D. V. Fedorov, and E. Garrido, Rev. Mod. Phys. 76, 215 (2004).

[39] K. Riisager, A. S. Jensen, and P. Møller, Nucl. Phys. A548, 393 (1992).
[40] J. Bartel, P. Quentin, M. Brack, C. Guet, and H. B. Hkansson, Nucl. Phys. A386, 79 (1982).

[41] E. Braaten and H.-W. Hammer, Phys. Rep. 428, 259 (2006).

[42] E. Garrido, D. V. Fedorov, H. O. U. Fynbo, and A. S. Jensen, Nucl. Phys. A781, 387 (2007).

[43] H. O. U. Fynbo, R. Álvarez-Rodríguez, A. S. Jensen, O. S. Kirsebom, D. V. Fedorov, and E. Garrido, Phys. Rev. C 79, 054009 (2009). 